\title{
Das Meer und Meereswesen in der Kunst und Quellen des Mittelalters. Vergleichende Analyse des Kupferstichs „Meerwunder” von Albrecht Dürer aus dem Jahr 1498
}

\author{
Przemysław WASZAK
}

\begin{abstract}
The article concentrates on showing elements present in culture, literature and art of the Middle Ages. These parts of mediaeval culture are discernible in Albrecht Dürer's graphic The Sea Monster, also known as the Wonder of the Sea. It was created by late Gothic-Renaissance author living at the turning point of both artistic epochs. The article consists of a description of the copperplate engraving, discussion on the contexts present in literature and mediaeval sources, analogies of artistic motifs and symbolic contents, meanings of the title which was already mentioned by Dürer.
\end{abstract}

Keywords: Albrecht Dürer, copperplate, sea monster, wonder of the sea, graphic, iconography, reception of mediaeval art, mediaeval sources

\section{Ziel, Gegenstand und Umfang des Beitrags}

Gegenstand der Analyse ist eine Grafik über ein weltliches Thema (Abb. 1). Sie gehört zum großen, etwa 100 Kupferstiche zählenden Ertrag des Nürnberger. Gleichzeitig ist sein Oeuvre, das Holzschnitte umfaßt, etwa doppelt so groß. ${ }^{2}$ Zahlreiche Werke von

1 Dieser Artikel ist eine überarbeitete und erweiterte Version des am 6. April 2019, an der Fakultät für Geschichte der Danziger Universität während der 5. Konferenz über die Meeres- und Flussgeschichte gehaltenen Vortrags. Zu den Forschungen zu diesem Thema trug das von der Fakultät für Geschichtswissenschaften der Nicolaus-Copernicus-Universität in Toruń zuerkannte Grant Nr. NH-1173 bei.

2 MURRAY, L. - MURRAY, P.: Sztuka Renesansu, Übersetzung J. Arszyńska, A. Mosingiewicz. Toruń - Wrocław 1999, S. 190.

3 GREBE, A.: Dürer: die Geschichte seines Rubms. Petersberg 2013, S. 175-193; Belege für Nachahmungen: STRAUSS, W. L.: Sixteenth century German artists: Albrecht Dürer, (The illustrated Bartsch 10). New York 1980, S. 158-159.
Dürer sind bereits zu seinen Lebzeiten Gegenstand von Nachahmungen und Kopien geworden. Das Meerwunder wurde von Wenzel von Olmütz schon im Jahr der Entstehung des Originals kopiert. ${ }^{3}$ Die zu besprechende Grafik gehört zu den bekannteren und häufig, auch in Monographien über den künstlerischen Ertrag Albrecht Dürers (1471-1528) diskutierten $^{4}$ und wird gern als Illustration verwendet. Sie

4 Unter anderem: DOLLMAYR, H.: Albrecht Duerers Meerwunder. In: Jabrbuch der Kunsthistorischen Sammlungen des Allerböchsten Kaiserbauses [ab 1919 Jahrbuch der Kunsthistorischen Sammlungen in Wien], 20, 1899, Theil I: Abhandlungen, S. 1-4; LANGE, K.: Dürer's „Meerwunder“. In: Zeitschrift für bildende Kunst, 35, [=N.F. 11] 1900, S. 195-204; Dürer - das Meerwunder. Eds.: RECKERT, A. - ARDJAH, M. - BIEBER, S. Delmenhorst 2012; STRIEDER, P.: Antike Vorbilder für Dürers Kupferstich „Das Meerwunder“. In: Anzeiger des Germanischen Nationalmuseums, 1971-1972, S. 42-47; Bibliographie gesammelt teilweise in: HUTCHISON, J. C.: Albrecht Dürer: a Guide to Research. Washington, DC 2005. URL: https://epdf. tips/albrecht-durer-a-guide-to-research-garland-reference-library-of-the-humanities.html [Zugriff: 01.02.2019]. 
kann jedoch immer noch zu Versuchen verwendet werden, neue Analysen und Beobachtungen anzustellen. Der vorliegende Beitrag konzentriert sich auf die Darstellung der noch aus der Kultur, Literatur und Kunst des Mittelalters stammenden Elemente, die in der Grafik Meerwunder dieses vielseitigen, an der Schwelle der Spätgotik-Renaissance lebenden Künstlers zu erblicken sind.

Die Überlegungen, welche auf der ein maritimes Thema aufgreifenden Grafik basieren, zeigen die mittelalterliche Welt der Ungeheuer, Hybriden und Meeresmonster, welche schon lange vor der Wende des 15. Jahrhunderts, als der zu besprechende Kupferstich geschaffen wurde, aus Kunstwerken und aus mittelalterlichen Quellen bekannt waren. Aufgezeigt werden auch die in den künstlerischen Abhandlungen aus dieser Zeit enthaltenen Analogien. Von Meermonstern sprechen oft theologische Quellen, welche von Menschen geschrieben wurden, die mit dem Meer nicht vertraut waren, sondern von Chronisten oder Theologen, Intellektuellen und Mystikern, die die Welt des Großen Wassers nur von der gelehrten Lektüre gekannt haben.

Nach der Beschreibung des Kupferstichs werden von uns Bezüge der darin enthaltenen detaillierten und universellen künstlerischen Problematik zu geschriebenen Quellen nachverfolgt. Analysiert wird die Rolle von Meeresmonstern in der Mystik des 14. Jahrhunderts. Wir versuchen Parallelen sowie Herkunft und Funktion sowohl bildlicher als auch ikonographischer Motive aufzuzeigen. In den Forschungen werden interdisziplinäre vergleichende Methoden eingesetzt, die Kunstwerke, geschriebene Quellen und literarische Texte umfassen.

\section{Beschreibung der Grafik}

Der Kupferstich wurde auf einem 25,1 x 18,7 cm großen Papierblatt geprägt. Im kompositorischen Zentrum der Grafik wurden zwei nackte Gestalten präsentiert: die einer Frau, welche von einem Hybrid mit Merkmalen eines Mannes und eines Fisches getragen wird. Die Kreatur bewegt sich frei auf der Wasseroberfläche, dringt fast nicht ins Wasser

\footnotetext{
5 Vergleiche: STRIEDER, P.: Dürer. Königstein im Taunus 1981, S. 186; mit Beiträgen von: GOLDBERG, G.: Zum technischen Befund von Albert Dürer „Vier Aposteln“;
}

ein, mit einer schnellen Bewegung wühlt er die Flussfläche auf, was Dürer durch sporadische aber dynamische, multidirektionale wellige Schraffuren erreicht. Oberhalb der zentralen Gruppe von Gestalten werden die ruhigen horizontalen Schraffuren gedrängter, und geben so die unerschütterliche Natur der Tiefe wider. In der Ferne, etwa auf halber Höhe des Bildes, ist ein zweites, vorwiegend mit kleinen Bäumen und Schilf bewachsenes Flußufer zu sehen. Auf die weiße Landzunge, die wie ein Laufsteg in die Wasserfläche ragt, läuft schnell ein älterer Mann, mit dramatisch nach oben gehobenen beiden Armen mit gespreizten Händen. Er zeichnet sich durch eine türkische Kopfbedeckung und einen von der schnellen Bewegung aufgewirbelten, orientalischen Mantel mit einem angeschnallten Säbel aus. Mit der bekleideten Gestalt stehen drei nackte Gestalten im Kontrast, die schwimmen, baden, sich ausruhen bzw. auf das Ufer gehen, und die bis auf eine eigentlich am Geschehen nicht teilnehmen, aus der theatralisch-phantastischen Aktion des Bildes entfremdet sind. Zugegebenermaßen läßt die spürbare Erregung dieser Gruppe auch eine andere Interpretation zu. ${ }^{5}$ Im Hintergrund sind hohe Mauern mit einem hervortretenden, runden Turm und einem Tor mit Zugbrücke zu sehen. Die Bauten sind mit sehr hohen, steilen Pult- bzw. Satteldächern gedeckt. Oberhalb, auf einer Böschung, steil wie ein Kliff, ist eine weitere, kleinere Burg mit drei zylindrischen Akzenten der Wachtürme zu sehen, wovon die erhabene bis zum oberen Rand des Bildfeldes reicht. Am rechten Rand, auf der halben Höhe der Grafik, wahrscheinlich schon auf der hohen See fährt - von der Heckseite gesehen - ein Schiff unter vollen Segeln. Der ruhige Himmel ist zur Hälfte mit Schraffuren bedeckt, die an Gestaltung der Wasserfläche erinnern, die andere Hälfte dagegen ist von einer majestätischen - größtenteils ohne Schraffuren - weißen Wolke verzogen. Der Künstler hat genauso großartig die zarten, breiten, stellenweise zusammengedrängten Falten der kleinen Fragmente einer Drapierung wiedergeben, die die Beine der Figur umwickelt, und von den Wellen getragen und vom Wind verweht wird. Erwähnenswert ist, dass Dürer eher ein Ufer eines Flusses, der ins Meer

HARNEST, J.: Dürer und die Perspektive; MENDE, M.: Aus Schriften Dürers: Aus Schriften über Dürer. 
Abb. 1: Albrecht Dürer (1471-1528), Das Meerwunder, 1498, Kupferstich, Blattgrösse: $25.1 \times 18.7 \mathrm{~cm}$. Photo: ETH-Bibliothek Zürich, Graphische Sammlung / D 12872 / Public Domain Mark 1.0, bttps:/ / doi.org/10.16903/ ethz-grs-D_012872

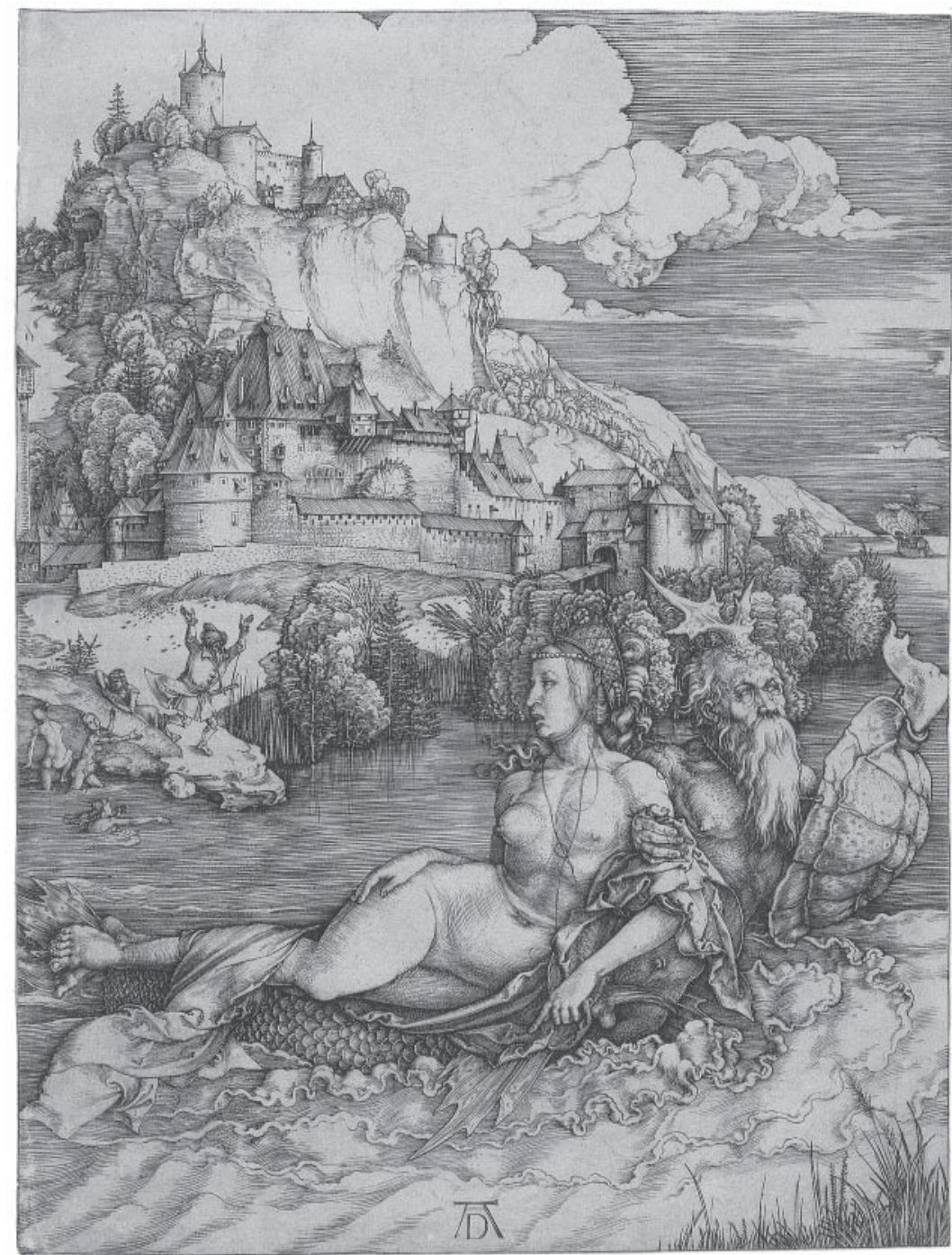

fließt, als ein Meeresufer auf der Grafik dargestellt hat, denn auf dem zweiten, fragmentarisch dargestellten Ufer, das dem Betrachter am nächsten liegt, ein Grasbüschel sichtbar ist. Das im Vordergrund dargestellte Grasbüschel kann in seiner Funktion als Motiv betrachtet werden, das dem Repoussoir ähnlich ist. Es ist buchstäblich ein ,zurücktreibendes" Objekt, das gleichzeitig der Komposition einen größeren Tiefeneindruck verleiht. ${ }^{6}$ An spätgotischen Tafelgemälden befand es sich oft in der rechten unteren Ecke in Form eines Stillebens, zum Beispiel eine Vase mit Blumen. In der Mitte, am unteren Bildrand befindet sich eine charakteristische Signatur - das Monogramm des Stechers.

Dürer präsentierte hier einen halbliegenden weiblichen Akt. Er zeigte das Modell in einer komplizierten, einstudierten und durch seine Multidirektionalität dynamischen Pose. Mit einer gewissen Unnatürlichkeit der Haltung ist eine ausgesuchte, verzierte Kopfbedeckung und die beeindruckend und mit Gefühl gestaltete Frisur einer

6 Stownik terminów, Sztuka świata, Bd. 18. Warszawa 2013, S. 192. 
von den Wellen getragenen Frau gut abgestimmt. Die Wiedergabe einer nackten, liegenden Gestalt war eine Neuheit in der damaligen Grafik, und die Darstellung selbst vereint in sich spätgotische und Renaissance-Merkmale. ${ }^{7}$

Die Grafik trägt genau dasselbe Datum, wie die bekannte Tafelmalerei: Das Ölgemälde Selbstbildnis mit Landschaft, das sich in den Sammlungen des Prado Museums in Madrid befindet. Dem selbstsicheren, reich gekleidete Mann, der auf dem Bild abgebildet wurde, mangelt es nicht an psychologischer Tiefe, und die Wiedergabe bestimmter Elemente, unter anderem der Kleidung weist Merkmale der künstlerischen Entschlossenheit und Virtuosität auf. Dank diesem Selbstporträt haben wir einen Beleg dafür, wie der Nürnberger im Entstehungsjahr des Meerwunders ausgesehen hat, und wie er sich selbst geben, bzw. wie er wahrgenommen werden wollte. Zum Zeitpunkt der Entstehung des Kupferstichs war er etwa 27 Jahre alt. Der zu besprechende Stich entstand nur wenige Jahre nach der Rückkehr des Künstlers von seiner ersten Italienreise nach Venedig, in der Zeit, als der Künstler sich zunehmend für die Problematik der plastischen Darstellung des Aktes zu interessieren begann. ${ }^{8}$

Zur Entstehungszeit des Meerwunders hatte die Kupferstichtechnologie bereits ein halbes Jahrhundert intensiver Entwicklung hinter sich. Sie wurde nämlich in den dreißiger Jahren des 15. Jahrhunderts erfunden. ${ }^{9}$ Der Kupferstich gehört zu den grafischen Tiefdrucktechniken. Seine Anfertigung verlangte vom Stecher beträchtliche Fertigkeiten ab. Diese frühe Tiefdrucktechnik macht eine detaillierte, trockene, dünne, zarte Linie möglich. Die so entstandene Zeichnung unterscheidet sich von der, die mit

HÜTT, W.: Niemieckie malarstwo i grafika późnego gotyku i renesansu, polnische Übersetzung von S. BLAUT. Warszawa 1985, S. 205.

8 STRIEDER, P.: Dürer. In: The Dictionary of Art, Bd. 9. Ed.: TURNER, J. New York 1998, S. 430.

9 SIKORSKA, J: Miedzioryt XV wieku i jego odrębność w sztuce późnego średniowiecza. Problemy badawcze. In: Biuletyn Historii Sžtuki, 70, 2008, Nr. 1-2, S. 115.

${ }^{10}$ KREJČA, A.: Techniki sz̨tuk graficznych:podręcznik metod warsz̨tatowych $i$ historii grafiki artystycznej, aus dem Deutschen übersetzt der Holzschnitttechnik geschaffen wird, die zum Hochdruck gehört, der in Europa etwas früher, denn gegen Ende des 14. Jahrhunderts zur Anwendung kam. Der Holzschnitt machte auch eine Evolution durch - angefangen von der rohen, unkomplizierten Linearität. ${ }^{10}$ In dieser Grafik nutzte Dürer Kupfersticheigenschaften gekonnt aus. Sie übertrifft Stiche, die sein Werk nachahmen.

\section{Literarische Quellen und ikonographische Kontexte}

Dies, was auf dem Stich gezeigt wurde, kann mit einer Passage aus einer frühen, vom Anfang des zwölften Jahrhunderts stammenden, geschriebenen Quellen über Techniken und Ästhetik der romanischen Kunst verglichen werden: „Im Hintergrund [...] zeichne Kreise und Zweige, und daran Blüten und Blätter [...] In die Kreise kannst du hier und da auch kleine Tiere, Vögel, Gewürm und nackte Gestalten einfügen...". ${ }^{11}$ Das angeführte Zitat ist ein Fragment des 21. Kapitels des zweiten Buches der bekannten Abhandlung - des Werkstatthandbuches Diversarum artium schedula sive de diversis artibus libri III vom Mönch Theophilus, bekannt auch als Rogerus von Helmarshausen (circa 1080 - nach 1125). Das Kapitel heißt: Über das Verzieren der Glasmalerei. ${ }^{12}$ Es kann auf die so dargestellte Natur bezogen werden - auf dem Glas - im Mikro- und in der Grafik Dürers im Makromaßstab, und auf das Vorhandensein von nackten Gestalten, die den Stich dekorieren und abwechslungsreicher machen sollten. Ähnlichkeiten sind präsent in den Fragen der grafischen Gestaltung des Dargestellten und in allgemeinen Rahmenbedingungen der Kompositionsprinzipien.

von A. DULEWICZ. Warszawa 1984, S. 23, 24, 27, 30, 65, 67, 68, 70 .

11 THEOPHILUS: Schedula diversarum artium, nach: BIEDERMANN, G., ROTH, K.: Schatzkammer Steiermark. Graz - Wien - Köln 1992, S. 45.

12 THEOPHILUS PRESBYTER: Diversarum artium schedula: średniowieczny zbiór przepisów o sztukach rozmaitych [Mittelalterliche Sammlung von Vorschriften über verschiedene Künste], Übersetzt aus dem Lateinischen und bearb. von S. KOBIELUS; Abbildungen, Einbandentwurf und Titelseite B. KULESZA-DAMAZIAK. Kraków 1998, S. 45. 
Ich möchte hier ein viel späteres Zitat anführen, das auch den Verlauf des kreativen Prozesses verrät. Die Aufzeichnung stammt aus den Tagebüchern des Malers Jakob Gauermann (1773-1843), genau aus dem Jahr 1838, und wurde 17 Jahre nach der Veröffentlichung der Autobiographie des Künstlers und 5 Jahre vor seinem Tod geschrieben. ${ }^{13}$ Es lohnt sich, nach dem man das Geschriebene mit der Grafik verglichen hat, zu fragen, ob sich seit der Dürerzeit bis zur Zeit der industriellen Revolution so viel in Bezug auf Prinzipien des künstlerischen Schaffens geändert hat: „Der Mahler muß nach seiner Idee eine Scize entwerfen und solche solange wenden und drehen und verbessern, bis er mit ihr zufrieden ist. Nach diesem sucht er in der Natur Studien auf, die zu seinem Entwurf behilflich sein können“'14. Die Zitate bestätigen die doppelte Verankerung eines Kunstwerks: sowohl in der Natur als auch im reichen Vorstellungsvermögen des Künstlers. Gauermann betont die Rolle der ausdauernden Arbeit eines Schöpfers. Nach ihm beginnt der Maler mit der Idee, mit dem Konzept des Kunstwerks, um erst dann zur Welt der Natur zu greifen, um die künstlerische Kreation, die bereits in seinem Kopf stattgefunden hat, lediglich nur noch zu vervollständigen und in einer ersten Fassung zu Papier übertragen wurde. Jakob Gauermann gehörte zur Familie der österreichischen Künstler. ${ }^{15}$ Er selbst war Kupferstecher und Maler. Er entwickelte seinen Stil eines Landschaftsmalers, indem er ,... nunmehr zu einem gesunden, auf eigener Naturbeobachtung basierenden Landschaftsrealismus überging". ${ }^{16}$ Seine Gabe für seine - zugegebenermaßen selten entstehenden - Landschaften wurde gut bewertet. ${ }^{17}$ So gibt es einige Parallelen zu

${ }^{13}$ GAUERMANN, J.: Kurze Lebensbeschreibung des Malers und Kupferstechers Jakob Gauermann, von ihm selbst verfaßt. In: Morgenblatt für gebildete Stände / Kunstblatt, 2, 1821, Nr. 57, 16. Juli 1821, S. 225-228; KRUG, W.: Gauermann. In: Allgemeines Künstlerlexikon: die bildenden Künstler aller Zeiten und Völker, Bd. 50. München 2006, S. 210.

${ }^{14}$ GAUERMANN, J.: Tagebücher. 1838 nach: BIEDERMANN/ ROTH (wie Anm. 11), S. 95.

${ }^{15}$ KRUG 2006 (wie Anm. 13), S. 208-211.

${ }^{16}$ MATĚJČEK, A.: Gauermann, Jacob. In: Allgemeines Lexikon der bildenden Künstler von der Antike bis zur Gegenwart, Bd. 13. Ed.: THIEME, U. Leipzig 1920, S. 265. der künstlerischen Erscheinung des Nürnberger, für den „Natur Meisterin der Schönheit war“..$^{18}$

Das Meer und die als eine Frucht der Phantasie und Lektüre entstandenen Vorstellungen über seine Bewohner waren auch bei Theologen, Schriftsteller gegenwärtig, die wahrscheinlich nie das Meer mit ihren eigenen Augen gesehen haben. Nicht nur fantastische, aber reale Meerestiere erweckten Grauen, wie wir dies dem Bericht des Chronisten Raoul Glaber (circa 985-1047) entnehmen können, der einen Wal erwähnt, dessen Erscheinen dem Unheil vorausgehen sollte. ${ }^{19}$ Ähnliche Gefühle spiegelt die Illumination De Balena (Über Wale) aus dem anglonormannischen Bestiarium von Ashmole vom Ende des zwölften Jahrhunderts wider, die Segler auf dem bewegten Meer zeigt, und darunter einen Wal, der zwei Thunfische verschlingt. Der Wal symbolisierte auch Tod, den Übergang in den Abgrund. ${ }^{20}$

Im 14. Jahrhunderts schrieb der hervorragende Vertreter der dominikanischen rheinländischen Mystik Heinrich Seuse (circa 1295-1366) Worte, die für Wahrnehmung von Meerestieren von Bedeutung sind. Er hat sie in seiner Abhandlung Horologium Sapientiae [Stundenbuch der Weisheit] formuliert, die für Latein beherrschenden fratres docti-Gelehrte Brüder, Predigerbrüder, Dominikaner bestimmt war. Das erste Zitat knüpft direkt an das Aussehen der maritimen Hybriden an, um dann gleich ihre moralische Interpretation zu geben: „Was du in der Vision außerdem gesehen hast: Die reiche Menge an Tieren, die erschienen wie Meeresmonster in Menschengestalt [Unterstreichung - PW], sind weltliche Herzen, die unter einem geistlichen Äußern versteckt sind, äußerlich eine ehrenvolle Erscheinung

${ }^{17}$ GAUERMANN 1821 (wie Anm. 13), S. 227 (Kommentar von Seufert).

${ }^{18}$ KOBIELUS, S.: Pojęcie piękna w pismach Albrechta Dürera. In: KOBIELUS, S.: Dzieło sztuki - dzieło wiary: przez widzialne do niewidzialnego. Ząbki 2002, S. 21.

${ }^{19}$ DAVY, M.-M.: Symbolika romańska (XII w.), übersetzt von K. WAKAR. Warszawa 2011, S. 62.

${ }^{20}$ Bodleian Library in Oxford, MS Ashmole 1511 fol. 86v. FOSSI, G.: Sztuka romańska i gotycka. Warszawa 2006, S. 77, Abb; KOBIELUS, S.: Bestiarium chrześsijańskie: zwierzetata w symbolice i interpretacji: starosiytność $i$ średniowiecæe. Warszawa 2002, S. 331, 332, Abb. 251. 
abgeben, aber innerlich leer von Gnade und Andacht sind. Und indem sie sich so innerlich und äußerlich verschieden zeigen, sind sie freilich tierischen Monstern nicht unähnlich“" ${ }^{21}$ Wichtig ist auch ein zweites Fragment, das auf weitere mögliche Verwendung der fantastischen, fremden Meeresschöpfungen in der moralischen Belehrung und Didaktik verweist: „Er [Geistlicher] ist mit einer Ruinenstadt zu vergleichen, welche entweder plötzlich einstürzt oder allmählich zerfällt, bis sie endlich ganz untergeht. Ebenso ist er mit einem Meeresmonster zu vergleichen wegen seiner äußeren und inneren Entstellung [Unterstreichung - PW]. Denn ein wahrer und vollkommener Geistlicher muß folgende Bedingungen erfüllen: Er muß alle weltlichen Dinge verachten, allein in Liebe zu seinem Schöpfer brennen, immer heilige Meditationen und gute Werke pflegen“. ${ }^{22}$

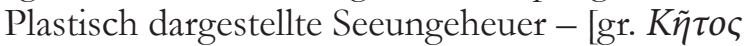
Kètos] sind bereits aus frühchristlichen Skulpturen und Sarkophagreliefs bekannt. Besonders erwähnenswert sind zwei Figuren aus Marmor, die Geschichte des alttestamentlichen Propheten Jonas zeigen, und sich in der Sammlung des Cleveland Museum of Art befinden. Sie stammen aus der zweiten Hälfte des dritten Jahrhunderts aus dem Osten des römischen Reiches, aus Kleinasien. Sie hatten wahrscheinlich eine sepulkrale Bestimmung. ${ }^{23}$ Auch ihnen wurden hybridische Formen verliehen. Das Ungeheuer hat Pfoten, Ohren, Schnauze eines Wolfes und Schwanzflosse eines großen Fisches. Ähnlich der Grafik von Dürer wurde hier der symbolische Übergang vom Abgrund zur irdischen Welt dargestellt. Den Unterschied machen gut erkennbare christologische Bezüge aus. Das Verschlucken und dann Rettung Jonas aus dem Rachen eines Wales verkündet in der altchristlichen Skulptur die Auferstehung Jesu Christi, und hat somit die gleichen soteriologische Konnotationen. Dürer schöpfte natürlich nicht direkt aus jenen zeitlich und räumlich entlegenen Kunstwerken.

${ }^{21}$ HEINRICH SEUSE: Stundenbuch der Weisheit = Das ,Horologium sapientiae ", Übersetzt von S. FENTEN, Würzburg 2007, S. 43.

${ }^{22}$ Ibidem, S. 213.

${ }^{23}$ CORMACK, R.: Byzantine art. Oxford - New York 2000, S. 14,15, Abb. 7.
Die Tradition der Darstellung von hybridischen Ungeheuern ähnlich der urchristlichen Ungeheuer bestätigen die romanischen Reliefs. Erwähnenswert ist der flache, zoomorphe Fries von der Stiftskirche St. Servatius im sächsischen Quedlinburg, die 1129 eingeweiht wurde, mit den aus der Lombardei abgeleiteten Formen. Beachtenswert sind die mit dicken Schuppen bedeckten Leiber und lange, gewundene Schwänze von Drachen bzw. fantastischen Hybriden oder Monstern. Auf Verbreitung solcher Motive in der architektonischen Skulptur verweisen weitere deutsche Beispiele. Anzuführen seien darüber hinaus die dem Quedlinburger Fries nachempfundenen Ungeheuer mit schlangenartigen Schwänzen aus der entlegenen kleinpolnischen Stiftskirche St. Martin in Opatów aus dem zweiten Viertel des zwölften Jahrhunderts. ${ }^{24}$ Die als flaches Relief ausgeführten Seeungeheuer wurden auf der Treppe zur Schau gestellt, die zum frühmittelterlichen Graboratorium aus der Merowinger Zeit um circa 700 in Poitiers (Hypogée des Dunes/Hypogeum auf den Dünen) führt, mit dem Dekorprogramm vom Abt Mellebaudus. Im Rahmen eines zum Gebet für die Toten abgesonderten Raumes werden sie als Wesen bezeichnet, die sich auf die letzten Dinge eines Gläubigen beziehen. ${ }^{25}$ Die Entführung einer Frau aus der menschlichen Welt von einem Wesen, das nicht von dieser Welt ist, kann in der Grafik Dürers auch in eschatologischen und zugleich moralischen Kategorien gedeutet werden.

\section{Analogien künstlerischer Motive}

Bei der Diskussion des Umfangs des künstlerischen Motivschatzes, das in dem Kupferstich von Dürer enthalten ist, sei darauf hingewiesen, dass Elemente aus der Waldwelt aus anderen Kunstwerken der damaligen Kunst bekannt sind. Zahlreiche Hirschgeweihe von beträchtlicher Größe und Elchschaufeln wurden in der berühmten spätgotischen

${ }^{24}$ JARZEWICZ, J.: Opatów a Quedlinburg, czyli o wnioskach $\mathrm{z}$ analogii. In: Architektura romańska w Polsce. Nowe odkrycia $i$ interpretacje. Materiaty z sesji naukowej w Muzeum Poczatków Państwa Polskiego, Gniezno 9-11 kwietnia 2008 roku. Ed.: JANIAK, T. Gniezno 2009, S. 324, 326-327, 332, Abb. 2.

${ }^{25}$ SKUBISZEWSKI, P.: Sztuka Europy tacinskiej od VI do IX wieku, vom Französisch übersetzt von J. KUCZYŃSKA und E. ZWOLSKI. Lublin 2001, S. 66, 69-70, Abb. auf S. 67. 
Holzskulptur der St.-Georg-Gruppe eingesetzt, welche sich im Stockholmer Dom St. Nicolaus befindet. St. Georg kämpft mit dem Drachen, welcher Satan und alles Böse bedeutet. ${ }^{26}$ Die geschnitzte Skulptur, die zu den hervorragendsten ihrer Art im Ostseeraum gehört, entstand 1489, also etwas früher als die Grafik. Die monumentale Statue wird einem niederländischen Bildhauer, bzw. dem bekannten Schöpfer Bernt Notke (um 1435-1509) zugeschrieben, der hauptsächlich in Lübeck tätig war. ${ }^{27}$

Als Vorlagen, die zur Entstehung einer idealistischen, romantischen Landschaft mit einer wehrhaften Burg in der Grafik Dürers beigetragen haben, könnten zahlreiche, malerische Ansichten deutscher Städte mit einem Burgberg an einem Fluß dienen. Die endgültige Form entstand jedoch als künstlerische Kreation von Dürer. Ein gewisses Sich-Wiederholen künstlerischer Motive und Stilisierung von Vorlagen, die sich aus der Beobachtung der Landschaften ergeben, weisen Gemälde von Dürer auf, die er während seiner Italienreise mit Alpenüberquerung gemalt hat. Die steilen Felswände eines erhabenen Berges mit der Burg auf der Spitze, sowie die Art und Weise, wie die Befestigungen dargestellt werden, sind den Elementen der Landschaft der zu besprechenden Grafik ähnlich. Als Beispiel können wir hier Aquarellansichten der Burg Arco und Castello del Buonconsiglio von 1495 anfüh$\operatorname{ren}^{28}$, die früher als der besprochene Kupferstich entstanden sind.

In der Grafik Dürers wurden 14 konkrete Baumund Pflanzenarten erkannt. Im vordergründigen

\footnotetext{
${ }^{26} \mathrm{Zu}$ den naturalistischen Elementen der Skulptur aus der Stockholmer Kathedrale (Storkyrka): KAREOWSKA-KAMZOWA, A.: Der Drache des Nordens. Eine ikonographische Analyse der Stockholmer Darstellung des Kampfes des Heiligen Georgs von Bernt Notke. In: Kościót i sqtuka pobrze ża Battyku = Kirche und Kunst im Ostseeraum, Bd. 3. Ed.: MURAWSKA, D. Toruń 1998, S. 146, 154-155, 158-159, Abb. 1, 4.

${ }^{27}$ TÅNGEBERG, P.: Wabrheit und Mythos - Bernt Notke und die Stockholmer St.-Georgs-Gruppe. Studien zu einem Hauptwerk. niederländischer Bildschnitzerei. Ostfildern 2009, passim.

${ }^{28}$ Abbildungen: ZUFFI, S.: Dürer: pasja i kunszt. Übersetzung: T. ŁOZINSKA. Warszawa 1998, Abb. auf S. 76-77.
}

Grasbüschel ist Plantago maior (Großer Wegerich) und Rumex acetosella (Kleiner Sauerampfer) zu sehen. ${ }^{29}$ Es handelt sich um allgemein bekannte und oft anzutreffende Pflanzen, die jeweils eine Heilpflanze und Unkraut sind. Dadurch ist das Werk Dürers nicht nur eine Art Illustration für ein märchenhaftes Bestiarium, sondern auch ein illustratives Herbarium, dargestellt mit schwarz-weißen, grafischen Mitteln.

Eine in gewisser Weise ähnliche Frisur der in der Grafik dargestellten Frau erscheint auf einem etwas früheren, bekannten, idealistischen Porträt einer im Profil dargestellten jungen Frau, das von Sandro Botticelli (um 1444-1510) zum Ausgang des Quattrocento in den Jahren 1480-1485 gemalt wurde. Es wird im Städel Museum in Frankfurt am Main aufbewahrt.

\section{Titel und Inhalt der Darstellung}

Die vom Künstler verliehenen oder allgemein in der Literatur verwendeten Titel von Kunstwerken haben einen konventionellen Charakter und können den Empfänger irreführen. Der in der polnischen Wissenschaft verwendete Titel lautet Driwo morskie analog zum Original: Das Meerwunder. Der Titel der Grafik erscheint in einem der vielen Werken des geschriebenen Ertrages von Dürer - Reise in die Niederlande. ${ }^{30}$ In englischer Sprache wird die Grafik in ähnlicher Weise als Wonder of the Sea ${ }^{31}$ bezeichnet, doch häufiger tritt sie unter dem Titel The Sea Mons$\operatorname{ter}^{32}$, auf, d. h. ein Meeresungeheuer, also genauso wie es Giorgio Vasari (1511-1574) beschrieben hat:

${ }^{29}$ RECKERT - ARDJAH - BIEBER 2012 (wie Anm. 4), S. 38-39, Abb. auf S. 39.

30 Albrecht Dürer jako pisarz i teoretyk særtuki [Auswahl, Einleitung, Übersetzung, Kommentar J. BIAŁOSTOCKI]. Wrocław 1956, S. 61.

${ }^{31}$ CARL, K.: Albrecht Dürer. Parkstone International 2016, S. 70. URL: search.ebscohost.com/login.aspx?direct $=$ true\&db $=\mathrm{n}$ lebk\&AN=906239\&lang $=$ pl\&site $=$ ehost-live \&scope $=$ site; [Zugriff 05. 02. 2019]; CHARLES, V.: Dürer. Parkstone International, 2011, S. 186. URL: search.ebscohost.com/ login.aspx?direct $=$ true \&db $=$ nlebk\&AN $=416855 \&$ lang $=-$ pl\&site $=$ ehost-live\&scope $=$ site. [Zugriff 05. 02. 2019].

${ }^{32}$ Zum Beispiel PANOFSKY, E.: The life and art of Albrecht Dürer. Princeton - New Jersey 1971, S. 73. 
Mostro Marino. ${ }^{33}$ Eine ähnliche Bezeichnung findet sich in der Übersetzung des Werks von Seuse ins Deutsche: Das Meeresmonster. In der lateinischen Sprache wird Seeungeheuer und Meerwunder mit den Wörtern monstrum marinum wiedergeben. ${ }^{34}$ Der Begriff Meeresmonster erscheint in modernen und historischen Wörterbüchern der deutschen Sprache eigentlich kaum, Meermunder dagegen ja. Dieses Wort kann Sirenen bezeichnen, also Entsprechungen von Wasserhybriden, die dem dargestellten Triton ähneln, also außergewöhnliche, märchenhafte Kreaturen, die gleichzeitig äußerst selten sind. ${ }^{35}$ Dürer hat zur Veranschaulichung zahlreicher realer, fantastischer oder halb phantastischer Kreaturen beigetragen, die biblische, hagiographische und mythologische Konnotationen haben. Seine Gemälde und Grafiken sind geradezu Illustrationen von mittelalterlichen Bestiarien.

\section{Die Symbolik}

Die Entführte hält mit starker Hand eine Kreatur mit einem menschlichen, muskulösen Torso, einem langen Bart, senilen Zügen, aus deren Kopf und der Schildkrötenschale ein kleines Geweih herauswächst. Das Geweih ist ein Motiv mit polarer Symbolik. Es ist einerseits eine Ankündigung des Kreuzes, kann aber auch den Satan bezeichnen. ${ }^{36}$ Das Meerwunder hat anstelle der Beine eine monolithische, mit einem dicken, breiteflächigen Schuppen bedeckte Form,

\footnotetext{
${ }^{33}$ SCHOCH, R.: Das Meerwunder (Kat. Nr. 21). In: Albrecht Dürer: das druckgraphische Werk, Bd. 1, Kupferstiche, Eisenradierungen und Kaltnadelblätter. Bearb. von R. SCHOCH, M. MENDE und A. SCHERBAUMS. München 2001, S. 74, Anm. 4.

${ }^{34}$ URL: http://www.woerterbuchnetz.de/cgi-bin/WBNetz/ startGlobalSearch.tcl? stichwort=Meerwunder [Zugriff 25.01.2019]. (Deutsches Wörterbuch von Jacob und Wilhelm GRIMM. 16 Bde. in 32 Teilbänden. Leipzig 1854-1961. Quellenverzeichnis Leipzig 1971. Online-Version vom 25.01.2019).

${ }^{35}$ URL: http://www.woerterbuchnetz.de/cgi-bin/WBNetz/ startGlobalSearch.tcl? stichwort=Meerwunder [Zugriff 25.01.2019] (Grammatisch-Kritisches Wörterbuch der Hochdeutschen Mundart).

${ }^{36}$ KOBIELUS 2002 (wie Anm. 20), S. 391-393.

${ }^{37}$ CIRLOT J. E.: Stownik symboli. Übersetzt von I. KANIA.
}

die mit der Flosse endet. Sie hat eine der Flosse von Triton ähnliche Form bzw. eine auch schon im Frühmittelalter populäre Darstellung der Sirene mit dem Fischschwanz. ${ }^{37}$ Erwähnenswert ist, dass Sirenen in Ansichten aus der Antike und zu Beginn des Mittelalters eine zur Hälfte Vogelform haben. ${ }^{38}$ Die Dürersche Gestalt kann in der Wildheit seiner tierischen Hälfte an einen Zentauren erinnern. ${ }^{39}$

Das dargestellte Meerwunder schützt sich mit dem Carapax - dem Rückenpanzer - wahrscheinlich einer großen Seeschildkröte. Dieser natürliche Panzer dient als Schild, wie dies bei den antiken griechischen Kriegern der Fall war, wie man im Mittelalter glaubte. ${ }^{40}$ Der Triton schützt sich damit, als würde er einen nicht in der Grafik dargestellten Feind angreifen. Nebenbei bemerkt, Dürer sammelte Schildkrötenschalen und wußte sie zu schätzten. Eine davon ließ er, als er später in Antwerpen während seiner Reise in die Niederlande zwischen 1520 und 1521 weilte, nach Nürnberg schicken. Gleichzeitig betonte er die beträchtliche Größe des abzuschickenden Schildkrötenpanzers. ${ }^{41}$

Fast ein Jahrhundert jünger als die Grafik ist das umfangreiche und einflussreiche Werk von Cesare Ripa (1555-1622) Iconologia. Es wurde erstmals 1593 verlegt. Ein Zeichen eines Phlegmatikers ist seiner Meinung nach das Wasser. Die auf dem Holzschnitt dargestellte charakteristische Figur dieses Temperaments wird von einer mittelgroßen Schildkröte begleitet. ${ }^{42}$ Aus der reichen Symbolik der Schildkröte

Kraków 2012, S. 398-399, Abb. auf S. 398-399 (Stichwort: Syrena/Sirene).

38 Stownik kultury antycznej: Grecja, Rzym. Ed.: WINNICZUK, L. [Autoren: M. FREYD et al.]. Warszawa 1986, S. 412 (Syreny/ Sirenen), 433 (Tryton/Triton).

${ }^{39}$ Oder Onokéntauros - der als Hybrid beschrieben wird, bestehend aus menschlichem Oberkörper und Eselrumpf: Fiøjologi i Aviarium: śedniowieczne traktaty o symbolice zwierzat. Übersetzt und bearb. von S. KOBIELUS, Hrsg. von E. PRZYBYZ. Kraków 2005, S. 48-49 (Fizjolog Bls, XII, O syrenach).

${ }^{40}$ KOBIELUS 2002 (wie Anm. 20), S. 357.

${ }^{41}$ Zum Beispiel Albrecht Dürer jako pisarz i teoretyk sztuki 1956 (wie Anm. 30), S. 81, 83.

42 RIPA, C.: Ikonologia. Übersetzt von I. KANIA. Kraków 2002, S. 189, Abb. auf S. 189. 
kann man auf den Bezug auf die Göttin Venus in der Antike und auf Vitalität und sogar Unsterblichkeit verweisen. Im mittelalterlichen Gedankengut sowie in der Renaissance und später nahm die Symbolik der Schildkröte eine eher negative Konnotation an, die sich auf Sünde und Dämonen bezog. Ihr wurden jedoch auch apotropäische, das Unheil oder Dämonen abwendende Funktionen zugeschrieben. ${ }^{43}$ Auf dem Kupferstich ist jedoch nur der gewaltige, ausdrucksstark und plastisch wiedergegebene Panzer sichtbar.

Man sah in der zu besprechenden Darstellung einen Moment, in dem ein von der Liebe erfülltes Wesen eine schöne irdische Frau in seine Welt entführt. ${ }^{44}$ Man findet in dieser Darstellung den Seegott Glaucus wieder, den Sohn von Poseidon, der Syme, Bewohnerin einer der Inseln in der Nähe der Küste von Kleinasien, entführt. ${ }^{45}$ Maciej Bóbr führt noch die in der Literatur funktionierende Möglichkeit an, dass hier die Entführung der Danaide Amymone also einer Tochter von Danaos durch Triton dargestellt wird, die dann von Poseidon, dem Vater von Triton, einer der Meeresgottheiten, gerettet wird. Meines Erachtens verweist Maciej Bóbr eher auf eine gewisse Interpretationsfreiheit, und die Möglichkeit der fehlenden Einbettung dieser im allgemeinen Sinne zweifellos phantastischen Darstellung in den antiken Mythologien. ${ }^{46}$ Es gibt mehrere suggerierten Varianten mythologischer und legendärer Konnotationen für dieses Werk. So wurden die Entführung der langobardische Königin Theodolinde, die Flucht der Schwester der Dido, Anna Perenna vor Aeneas genannt, und man legte nahe, dass die Grafik sich auf Metamorphosen oder die Mythensammlung Fasti von Ovid anlehnt. ${ }^{47}$ Erwin Panofsky und früher Konrad Lange verweisen auf die Geschichte von Poggio Bracciolini (1380-1459) oder eine anderen, der ihr ähnlich ist. ${ }^{48}$ Vorschläge, der Gestalt einen Namen

\footnotetext{
${ }^{43}$ KRETSCHMER, H.: Lexikon der Symbole und Attribute in der Kunst. Stuttgart 2014, S. 364-365; Kobielus 2002 (wie Anm. 20), S. 357.

${ }^{44}$ BIAŁOSTOCKI, J.: Sztuka XV wieku. Od Parlerów do Dürera. Übersetzt von G. PRZEWLOCKI, Hrsg. von A. ZIEMBA. Warszawa 2010, S. 318.

${ }^{45}$ Bildindex der Kunst \& Architektur, URL: https://www. bildindex.de/document/obj05091674?part=0\&medium= [Zugriff 28. 01. 2019].
}

zu geben und eine literarische Quelle für die Grafik zu bestimmen, sind in der Literatur noch ein paar mehr. Darin eine auf der griechischen Mythologie basierenden Identifikation als Entführung von Perimele durch Acheloos. ${ }^{49}$

Alle Interpretationen versuchen, die in der rätselhaften Grafik dargestellten Figuren aus der Namenslosigkeit zu erheben. Der Triton ist sowohl eine konkrete Figur: der mythische Sohn von Poseidon und Amphitrite als auch ein in zahlreichen Kunstwerken in Form von Tritonen popularisiertes künstlerisches Motiv. Auf der Grafik fehlt das charakteristische Attribut - die Muschel des Herolds. Dies machte jedoch die modernisierte Thematik der Darstellung nicht erforderlich, und die freie Interpretation von aus anderen Kunstwerken und aus der Literatur entlehnten Vorlagen. Die Forscher sind sich uneinig hinsichtlich der Möglichkeit, konkrete mythologische Vorlagen oder Geschichten auszumachen, auf die sich die besprochene Grafik beziehen würde. ${ }^{50}$

Die auf dieser wertvollen Grafik dargestellte Szene kann in allgemeinen, aufgezeigten und oben analysierten Kategorien verstanden werden, aber auch in literarischen, legendären, bildlichen und quellenmäßigen Kontexten. Der Inhalt der Darstellung gehört zur imaginären Welt der Monster und Hybriden, die vom Künstler in Anlehnung an mündliche und schriftliche Überlieferungen, und künstlerisch verarbeitete Welt der Natur entnommen und von ihm komponiert wurden. Er sieht aus, wie aus mittelalterlichen und alten Geschichten über echte und fantastische Tiere entlehnt. Die Motive des Bildes sowie deren Fassung sind eingebettet in die Kultur und Tradition des Mittelalters. Menschen der Feder, Illuminatoren, Stecher der Gotikzeit schufen ihre eigene maritime Welt unabhängig von tatsäch-

\footnotetext{
${ }^{46}$ BÓBR, M.: Mistryowie grafiki europejskiej: od XV do XVIII wieku. Warszawa 2000, S. 52, Abb. 55 auf S. 51.

${ }^{47}$ HUTCHISON 2005 (wie Anm. 4), S. 28-29, 40.

${ }^{48}$ LANGE 1900 (wie Anm. 4), S. 201; PANOFSKY 1971 (wie Anm. 32), S. 73.

${ }^{49}$ HUTCHISON 2005 (wie Anm. 4), S. 28-29, 40, 81, 116.

${ }^{50}$ SCHOCH 2001 (wie Anm. 33), S. 74.
} 
lichen oder wahrscheinlichen Voraussetzungen. Sie wollten belehren, den Leser oder den Empfänger der Kunst rühren, eigene visuelle oder verbale Aussagen lebendiger und abwechslungsreicher gestalten. Sie schufen inhaltsreiche und für Empfänger und Käufer attraktive Bilder, sowohl in formaler als auch semantischer Hinsicht. Die Kupferstecher schöpften aus künstlerischen Leistungen anderer Bereiche künstlerischen Schaffens. Die Analyse der in der Abbildung dargestellten Welt zeigte eine grenzüberschreitende Gemeinschaft von Themen und Motiven, die in Kunstwerken verschiedener Techniken, Epochen und künstlerischer Regionen auftauchen. Kontexte, die auf den Kupferstich bezogen werden können, sowohl die früheren als auch viel späteren, belegen die zeitlose Bedeutung dieses Kunstwerks. Die angewandten formalen und ikonographischen Lösungen, Dialog und Symbiose von Phantasie und Realität ermöglichen einen neuen Einblick in die alte Kunst. Die Formen und Inhalte der Kunst von Dürer bilden einen Bezugspunkt für Analyse anderer Kunstwerke, Techniken und Künstlergestalten. Sie erlauben es, die Kunst weiterer Kreise und Zeiten besser zu verstehen. Die Elemente der Dürerschen dargestellten Welt sind in Bildern und Formen des Mittelalters verwurzelt, die den Schlüssel zum Verständnis der christlichen Kunst der späteren Kunstperioden bilden.

\title{
The Sea and Sea Creatures in the Art and Sources of the Middle Ages. Comparative Analysis of the Copperplate „Sea Monster“ by Albrecht Dürer Dated to the 1498
}

\author{
Résumé
}

The article concentrates on showing elements present in culture, literature and art of the Middle Ages. These parts of mediaeval culture are discernible in Albrecht Dürer's graphic The Sea Monster, also known as the Wonder of the Sea. It was created by late Gothic-Renaissance author living at the turning point of both artistic epochs. The article consists of a description of the copperplate engraving, discussion on the contexts present in literature and mediaeval sources, analogies of artistic motifs and symbolic contents, meanings of the title which was already mentioned by Dürer. The deliberations show the world of monsters, hybrids, sea-monsters known from sources long before the creation of this engraving. Depictions of hybrids and other mentioned creatures were widely used earlier for example in the Early Christian art, Merovingian art, Romanesque art. Examples from these epochs are quoted. Especially important are moralistic mentions of sea hybrids in the literary works of Heinrich Seu- se, a $14^{\text {th }}$ century prolific theologian and exponent of Rhineland mysticism. His statements can serve as a good example of commonly known practices. There are also discussed ways of perceiving art and its fantastic and fabulous world both in earlier examples and in the statements of a $19^{\text {th }}$ century artist. This comparison shows what is constant in art regardles of epoch and what changes during the long period of artistic development. The questions of relation of art to nature were discussed, too. The analysis of the world depicted on the print shows a community of plots and motifs which appear in the works of art of different techniques, epochs and artistic regions. Gothic man of letters and artists, for example illuminators, engravers created their own marine world. Their aim was to teach and instruct the reader, add colour to their visual works of art or to their verbal statements. Graphic artists draw their motifs from the artistic achievements of other fields of arts and knowledge. 